\title{
Addressing Challenges in Developing a Core Domain Set in Adherence Interventions in Rheumatology: A Report from the OMERACT-Adherence Group
}

\author{
Ayano Kelly, Susan J. Bartlett, Maarten P. de Wit (B, Dorcas E. Beaton, Therese Dawson, \\ Vicki Evans, Michael Gill, Geraldine Hassett, Lyn March, Marieke Scholte-Voshaar, \\ Jasvinder A. Singh, Allison Tong, Peter Tugwell, Peter Wong, and Kathleen Tymms
}

ABSTRACT. Objective. The OMERACT-Adherence meeting was convened to discuss the conceptual and methodological challenges in developing a core domain set (Adherence-CDS) for trials of interventions for medication adherence in rheumatology.

Methods. Forty participants from nine countries participated.

Results. Four ideas emerged: for adherence trials, the Adherence-CDS could include adherence and the condition-specific CDS; many factors affect adherence and are intervention targets, contextual factors, or outcome domains; adherence is a critical factor in drug trials; and standardized adherence measures are needed.

Conclusion. Despite the challenges, the meeting clarified an approach to developing an Adherence-CDS that complements existing OMERACT work and methodology. (First Release January 15 2019; J Rheumatol 2019;46:1202-6; doi:10.3899/jrheum.181078)

Key Indexing Terms:

TREATMENT ADHERENCE AND COMPLIANCE

OUTCOME ASSESSMENT (HEALTHCARE)

OMERACT

RHEUMATIC DISEASES

From the Canberra Rheumatology; College of Health and Medicine, Australian National University; Clear Vision Consulting; Discipline of Optometry, University of Canberra; Department of Rheumatology, Canberra Hospital, Canberra; Centre for Kidney Research, The Children's Hospital at Westmead; Dragon Claw (Web site for patients with rheumatic disease); Department of Rheumatology, Liverpool Hospital; Ingham Institute of Applied Medical Research; Department of Rheumatology, Royal North Shore Hospital; Institute of Bone and Joint Research, Kolling Institute of Medical Research; Northern Clinical School, and the School of Public Health, The University of Sydney, Sydney; Lord Street Specialist Centre, Port Macquarie; Mayo Hospital Specialist Centre, Taree; MidNorth Coast Arthritis Clinic; University of New South Wales Rural Clinical School, Coffs Harbour, Australia; Department of Medicine, McGill University; Centre for Outcomes Research and Evaluation, McGill University Health Centers, Montreal; Musculoskeletal Health and Outcomes Research, Li Ka Shing Knowledge Institute, St. Michael's Hospital; Institute for Work and Health; Department of Occupational Science and Occupational Therapy and the Institute of Health Policy Management and Evaluation, University of Toronto, Toronto, Ontario, Canada; Division of Rheumatology, Johns Hopkins School of Medicine, Baltimore, Maryland; Medicine Service, VA Medical Center, Birmingham; Department of Medicine, School of Medicine, and the Division of Epidemiology, School of Public Health, University of Alabama, Birmingham, Alabama, USA; Amsterdam University Medical Centre, Department of Medical Humanities, Amsterdam Public Health, Amsterdam; University of Twente, Department of Psychology, Health and Technology, Enschede, the Netherlands.

The work of OMERACT-Adherence to date has been supported by a 2018 Arthritis Australia project grant. JAS is a member of the executive of OMERACT, an organization that develops outcome measures in rheumatology and receives arms-length funding from 36 companies.

A. Kelly, FRACP, Australian National University, Canberra Rheumatology, and College of Health and Medicine, Australian National University, and Centre for Kidney Research, The Children's Hospital at Westmead; S.J. Bartlett, PhD, Department of Medicine, McGill University, and Centre for Outcomes Research and Evaluation, McGill University Health Centers, and Division of Rheumatology, Johns Hopkins School of Medicine; M.P. de
Wit, PhD, Amsterdam University Medical Centre, Department of Medical Humanities, Amsterdam Public Health, and OMERACT Patient Research Partner; D.E. Beaton, PhD, University of Toronto Musculoskeletal Health and Outcomes Research, Li Ka Shing Knowledge Institute, St. Michael's Hospital, and Institute for Work and Health, and Department of Occupational Science and Occupational Therapy, and the Institute of Health Policy Management and Evaluation, University of Toronto; T. Dawson, MPH, Lord Street Specialist Centre, and Mayo Hospital Specialist Centre; V. Evans, PhD, Clear Vision Consulting, and OMERACT Patient Research Partner, and Discipline of Optometry, University of Canberra; M. Gill, BA, Australian National University, and Dragon Claw, and OMERACT Patient Research Partner; G. Hassett, PhD, University of New South Wales, and Department of Rheumatology, Liverpool Hospital, and Ingham Institute of Applied Medical Research; L. March, PhD, Department of Rheumatology, Royal North Shore Hospital, and Institute of Bone and Joint Research, Kolling Institute of Medical Research, and Northern Clinical School, The University of Sydney; M. Scholte-Voshaar, MSc, Vrije Universiteit Amsterdam, and University of Twente, Department of Psychology, Health and Technology, Enschede, and OMERACT Patient Research Partner; J.A. Singh, MD, Medicine Service, VA Medical Center, and Department of Medicine, School of Medicine, University of Alabama, and Division of Epidemiology, School of Public Health, University of Alabama; A. Tong, PhD, Centre for Kidney Research, The Children's Hospital at Westmead, and University of Sydney, School of Public Health; P. Tugwell, MD, Division of Rheumatology, Department of Medicine, and School of Epidemiology and Public Health, Faculty of Medicine, University of Ottawa, and Clinical Epidemiology Program, Ottawa Hospital Research Institute; P. Wong, PhD, Mid-North Coast Arthritis Clinic, and University of New South Wales Rural Clinical School; K. Tymms, FRACP, Canberra Rheumatology, and College of Health and Medicine, Australian National University, and Department of Rheumatology, Canberra Hospital.

Address correspondence to Dr. A. Kelly, Canberra Rheumatology, Level 9 , 40 Marcus Clarke St., Canberra City, Canberra ACT 2601, Australia. E-mail: Ayano.Kelly@anu.edu.au

Accepted for publication November 26, 2018. 
Medication adherence is suboptimal in rheumatic diseases and has been reported to be as low as 10\% in gout and 30\% in rheumatoid arthritis (RA) $)^{1,2}$. Broadly, adherence is defined as "the extent to which a person's behavior - taking medication, following a diet, and/or executing lifestyle changes, corresponds with agreed recommendations from a health care provider"3. The Outcome Measures in Rheumatology Adherence Special Interest Group (OMERACT-Adherence Group) is currently developing a core domain set (CDS) for trials of interventions to improve medication adherence in rheumatology (Adherence-CDS). Our group includes patients, health professionals, and others in a project consisting of a systematic review, qualitative studies, a Delphi survey, and a consensus workshop ${ }^{4}$.

There are several trials of adherence interventions in rheumatology 5 . However, no group has examined what outcome domains should be measured in these trials, and those used currently are inconsistent and heterogeneous ${ }^{6}$. Historically, most CDS in OMERACT have been established for specific conditions. Developing a CDS that is focused on the intervention type rather than a specific condition requires careful thought. In addition, the work of this group is challenging because of the complexity of adherence. There are hundreds of determinants of nonadherence, and reviews of adherence interventions have shown that multifaceted, behavioral interventions are needed to address adherence and produce a substantial change ${ }^{7}$.

Developing a CDS for complex behavioral interventions that address adherence in rheumatic conditions does not easily fit with the existing OMERACT filter ${ }^{8,9}$. This report summarizes discussions during the OMERACT-Adherence Group meeting at OMERACT 2018 in Australia, which aimed to:

1. understand how an Adherence-CDS can be developed alongside existing condition-specific CDS;

2. review the candidate adherence-related domains from the work to date; and

3. modify the existing working plan.

\section{MATERIALS AND METHODS}

OMERACT-Adherence premeeting reading materials. Prior to the meeting, participants were provided with the OMERACT-Adherence protocol paper ${ }^{4}$, the European Society of Patient Adherence, COMpliance and Persistence Medication Adherence Reporting Guidelines (EMERGE) ${ }^{10}$, and the proposed meeting agenda.

Meeting presentations. The meeting commenced with presentations on the definition of medication adherence (including phases: initiation - when the patient takes the first dose of medication; implementation - extent to which a patient's actual dosing corresponds to the prescription; and persistence length of time between initiation and last dose) ${ }^{11}$, research plan ${ }^{4}$, and preliminary results of the systematic review and focus group studies. These studies will be reported separately; however, preliminary findings are provided below for background to the meeting discussion.

The systematic review examined adherence-related domains in existing randomized and nonrandomized trials of interventions to improve medication adherence in rheumatic conditions. Extracted domains included adherence and adherence-related domains (any domain related to adherence behavior). To date, the most common domains included medication adherence, concerns, knowledge, beliefs, and necessity. Preliminary findings from a nominal group technique study of patients and caregivers with RA, gout, and osteoporosis indicated that trust in doctor, medication effectiveness, medication side effects, doctor's knowledge, and disease knowledge were important factors influencing medication adherence. The Australian Capital Territory Health Human Research Ethics Committee (ETHLR.15.137) provided ethical approval and all participants provided written informed consent to publish the results of our study.

Meeting discussion. Attendees were provided with an exercise sheet (Figure 1) and a list of preliminary adherence-related domains. Smaller group discussions facilitated by OMERACT-Adherence co-chairs and group members (AK, SJB, MDW, TD, VE, MG, GH, MSV, KT) preceded a larger group discussion facilitated by AK, summarizing participants' perspectives and suggestions on a flip chart. OMERACT-Adherence Group member attendees contributed to this report.

\section{RESULTS}

Forty participants including patients, health professionals, researchers, industry, and regulators from 9 countries contributed to 4 main themes (Table 1):

1. An Adherence-CDS could include adherence and the condition-specific CDS. Meeting attendees discussed the Adherence-CDS using the PICOC framework (Patient/Intervention/Comparator/Outcome/Context; Figure 2). They suggested adding adherence as a domain to the condition-specific CDS. Although adherence was perceived as a domain itself, it was also considered an explanatory variable, process measure, or biomarker for the condition-specific CDS. One participant suggested that subdomains of adherence could address the phases of initiation, implementation, and persistence. Participants noted that there may be inadequate power or duration of followup to demonstrate changes in disease outcomes even if adherence is improved. For example, use of a comparator group also taking antirheumatic medications may reduce the magnitude of clinical benefit. There was considerable disagreement about whether adherence versus clinical outcomes should serve as the primary outcome. It was suggested that trials aiming to improve adherence in the real-world setting should only be conducted on medications with established efficacy and should be specified in the "Context" of the PICOC framework for the Adherence-CDS.

2. Factors from the systematic review and focus groups should be classified as intervention targets, contextual factors, or outcome domains. Participants noted that some adherence-related domains identified from the systematic review and focus groups were more accurately classified as targets of interventions or explanatory variables/contextual factors/process measures that influence adherence (e.g., medication knowledge). They provide insight into how adherence interventions can be conceptualized and designed, or could be used to develop a tool to predict adherence, and were suggested to be termed "adherence-related factors."

3. Adherence is a critical factor in drug trials. Drug trials

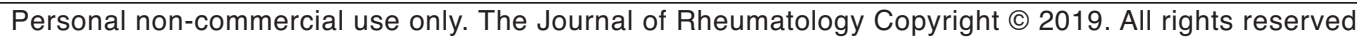




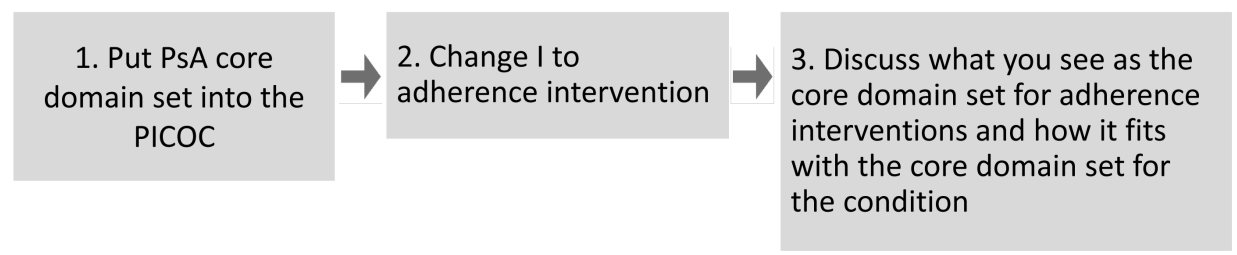

\section{Population:}

\section{Intervention:}

\section{Comparison:}

\section{Outcome:}

\section{Context:}

PsA core domain set (inner core):
MSK disease activity
Skin disease activity
Pain
Patient global
Physical function
Health related quality of life
Fatigue
Systemic inflammation

Figure 1. Exercise sheet for OMERACT-Adherence meeting discussion. OMERACT: Outcome Measures in Rheumatology; PsA: psoriatic arthritis; PICOC: Patient/Intervention/Comparator/Outcome/Context; MSK: musculoskeletal.

Table 1. Key recommendations from the OMERACT-Adherence workshop.

Key Recommendations

1. Adherence in the clinical setting is important to all, and better ways to measure the efficacy of trials of adherence interventions are needed.

2. There is value in determining the outcome domains to be measured in adherence trials prior to standardizing adherence measures.

3. The key outcome domains that may be in the core domain set for adherence trials are adherence in addition to the core domain set for the condition.

4. Using the condition-specific core domain set for adherence trials is difficult because of the lack of power, duration of followup, participant burden, and the comparator group used.

5. Our systematic review will evaluate the use of condition-specific core domains including medication toxicity/side effects in adherence trials.

6. We will determine which adherence-related factors are outcome domains versus targets of interventions or explanatory variables for improving adherence.

7. Adherence in drug trials and adherence measures are important independent areas of study.

OMERACT: Outcome Measures in Rheumatology.

(e.g., placebo-controlled trial for an osteoporosis medication) are different from adherence trials (e.g., randomized trial of intensive pharmacist support to address nonadherence). The OMERACT-Adherence Group is currently developing a CDS for adherence trials. Attendees discussed that in drug trials, adherence is an important contextual factor affecting the interpretation of clinical benefit and safety, although this is currently out of the scope of the OMERACT-Adherence work. There was agreement that the reasons for nonadherence in drug trials may differ from those in adherence trials, and therefore different studies would be required to investigate this topic.
Some attendees thought that nonadherence was not an issue in drug trials, although there is existing evidence to the contrary ${ }^{12}$.

4. Standardized adherence measures are needed in all trials. Participants recognized the need to standardize adherence measures in both adherence and drug trials. There was agreement that in trials, objective measures of adherence are essential, given the well-recognized bias associated with self-reports. Several individuals noted the potential role for measuring serum drug levels and use of technology such as micro-biosensors integrated into pills. The OMERACT-Adherence Group plans to develop a core outcome measurement 
Population

Intervention

Comparator

Outcome

Context
Adults with a rheumatic condition

(e.g. PsA)

Strategies aimed to improve medication adherence

(e.g. use of a decision aid)

Management as usual

Adherence plus condition-specific

CDS (e.g. PsA CDS)

Figure 2. Proposed OMERACT-Adherence CDS. OMERACT: Outcome Measures in Rheumatology; PsA: psoriatic arthritis; CDS: core domain set.

set after the CDS is established. However, a few participants proposed moving directly to standardizing adherence measures because they felt the OMERACT filter was difficult to apply to an adherence trial.

\section{DISCUSSION}

Forming consensus on what to measure in complex behavioral interventions that address medication adherence across rheumatic conditions is challenging, and the direct application of OMERACT Filter 2.1 ${ }^{9,10}$ is not straightforward. The suggestion to add adherence to the inner circle of a conditionspecific CDS (as being mandatory in an adherence trial) offers a potential solution. Adherence-related factors must be clearly classified as targets of interventions or explanatory variables for adherence, though some may be candidate outcome domains. Adherence as a contextual factor of drug trials, and consensus on standardized adherence measures, are important and require further investigation.

Participants who found it difficult to apply the OMERACT filter to an adherence trial did not see the value in reaching consensus on outcome domains and suggested proceeding immediately to standardization of adherence measures. However, similar to condition-specific CDS, an Adherence-CDS can reduce inconsistent reporting and reporting bias and promote measurement of outcomes that matter to patients ${ }^{10}$.

Clinical outcomes represented by the condition-specific CDS are infrequently reported domains of adherence trials, but are examples of outcomes that matter to patients and were highly valued in our focus groups. The importance of measuring medication side effects was demonstrated in a cohort study of kidney transplant recipients who had increased risk of malignancy with higher medication adherence $^{13}$. Making it mandatory to report the condition-specific CDS in all adherence trials could provide valuable information and progress in adherence research. Although discussions highlighted the difficulties of using the condition-specific CDS in adherence trials, limitations in power and duration of followup can also apply to drug trials for some clinical outcomes such as mortality.

Future steps need to address the interdependence of the condition-specific CDS with the Adherence-CDS and whether clinical outcomes are important and feasible to measure in all adherence trials. These activities have been prioritized to move forward. We will update the systematic review and assess whether existing adherence trials also include the condition-specific CDS, including reporting of medication side effects. Domains extracted from the systematic review and focus groups will be termed adherence-related factors, with further work to scrutinize what will be candidate domains for the Adherence-CDS. We plan to reconcile the disagreement regarding the primary outcomes in adherence trials (improved adherence vs clinical benefit).

Adherence is important in the clinical setting not only to patients and clinicians, but also to regulators and payers to ensure that patients maximize the potential health benefits from medications. As an increasing number of interventions are conducted to improve adherence in rheumatology, these need to measure relevant and consistent outcome domains. Key recommendations from the OMERACT-Adherence group following the workshop are summarized in Table 1.

\section{ACKNOWLEDGMENT}

The authors acknowledge the contribution of other members of the OMERACT-Adherence group who have contributed to the development, design, conduct, and analysis of the studies to date: Alexa Meara, Annica

$$
\text { Personal non-commercial use only. The Journal of Rheumatology Copyright (c) 2019. All rights reserved. }
$$


Barcenilla-Wong, Bart Van den Bemt, Beverley J. Shea, Catherine Hill, Christine Bailey, Clare O'Sullivan, Daniel Sumpton, Francois Nantel, Helen Keen, Irwin Lim, Karine Toupin-April, Lara Maxwell, Luke Crimston-Smith, Maria Suarez-Almazor, Marita Cross, Mary De Vera, Peter Cheung, Rani Sinnathurai, Renske Hebing, Rieke Alten, Robby Nieuwlaat, Robin Christensen, Sabrina Mai Nielson, Sean O’Neill, Stephen Hall, Willemina Campbell, and Yomei Shaw.

\section{REFERENCES}

1. De Vera MA, Marcotte G, Rai S, Galo JS, Bhole V. Medication adherence in gout: a systematic review. Arthritis Care Res 2014;66:1551-9.

2. van den Bemt BJ, Zwikker HE, van den Ende CH. Medication adherence in patients with rheumatoid arthritis: a critical appraisal of the existing literature. Expert Rev Clin Immunol 2012;8:337-51

3. Sabaté E. Adherence to long-term therapies: evidence for action. World Health Organization; 2003. [Internet. Accessed November 30, 2018.] Available from: www.who.int/chp/knowledge/ publications/adherence_full_report.pdf

4. Kelly A, Tong A, Tymms K, March L, Craig JC, De Vera M, et al; OMERACT-Adherence Group. Outcome Measures in Rheumatology - Interventions for medication adherence (OMERACT-Adherence) core domain set for trials of interventions for medication adherence in rheumatology: 5 phase study protocol. Trials 2018;19:204.

5. Galo JS, Mehat P, Rai SK, Avina-Zubieta A, De Vera MA. What are the effects of medication adherence interventions in rheumatic diseases: a systematic review. Ann Rheum Dis 2016;75:667-73.
6. Kelly A, Sumpton D, O’Sullivan C, Meara A, Nieuwlaat R, Tugwell $\mathrm{P}$, et al. Scope and consistency of adherence related outcomes in randomized controlled trials of interventions for improving medication adherence [abstract]. Arthritis Rheumatol 2017;69:1231.

7. Kardas P, Lewek P, Matyjaszczyk M. Determinants of patient adherence: a review of systematic reviews. Front Pharmacol 2013;4:91.

8. Boers M, Kirwan JR, Tugwell P, Beaton D, Bingham CO III, Conaghan PG, et al. The OMERACT Handbook. [Internet Accessed May 17, 2017.] Available from: https://omeract.org/resources

9. Boers M, Kirwan JR, Wells G, Beaton D, Gossec L, d'Agostino MA, et al. Developing core outcome measurement sets for clinical trials: OMERACT filter 2.0. J Clin Epidemiol 2014;67:745-53.

10. De Geest S, Zullig LL, Dunbar-Jacob J, Helmy R, Hughes DA, Wilson IB, et al. ESPACOMP Medication Adherence Reporting Guideline (EMERGE). Ann Intern Med 2018;169:30-5.

11. Vrijens B, De Geest S, Hughes DA, Przemyslaw K, Demonceau J, Ruppar T, et al; ABC Project Team. A new taxonomy for describing and defining adherence to medications. Br J Clin Pharmacol 2012;73:691-705.

12. Blaschke TF, Osterberg L, Vrijens B, Urquhart J. Adherence to medications: insights arising from studies on the unreliable link between prescribed and actual drug dosing histories. Annu Rev Pharmacol Toxicol 2012;52:275-301.

13. Yadav K, Vock DM, Matas AJ, Robiner WN, Nevins TE. Medication adherence is associated with an increased risk of cancer in kidney transplant recipients: a cohort study. Nephrol Dial Transplant 2019;34:364-70. 